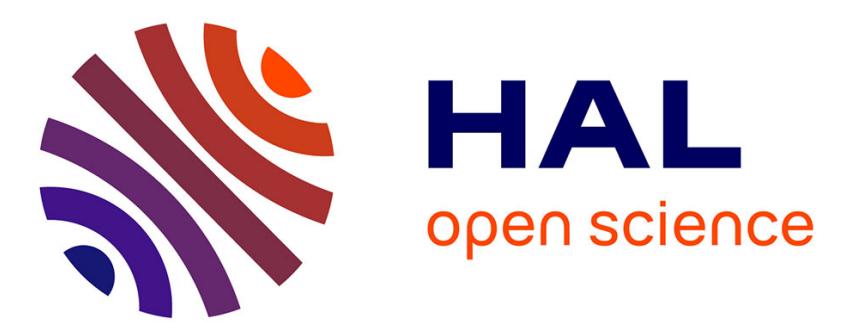

\title{
Identification of standing fronts in steady state fluid flows: exact and approximate solutions for propagating MHD modes
}

\author{
Filippo Pantellini, Léa Griton
}

\section{- To cite this version:}

Filippo Pantellini, Léa Griton. Identification of standing fronts in steady state fluid flows: exact and approximate solutions for propagating MHD modes. Astrophysics and Space Science, 2016, 361 (10), 10.1007/s10509-016-2921-y . hal-01382916

\section{HAL Id: hal-01382916 https: / hal.sorbonne-universite.fr/hal-01382916}

Submitted on 17 Oct 2016

HAL is a multi-disciplinary open access archive for the deposit and dissemination of scientific research documents, whether they are published or not. The documents may come from teaching and research institutions in France or abroad, or from public or private research centers.
L'archive ouverte pluridisciplinaire HAL, est destinée au dépôt et à la diffusion de documents scientifiques de niveau recherche, publiés ou non, émanant des établissements d'enseignement et de recherche français ou étrangers, des laboratoires publics ou privés. 


\title{
Identification of standing fronts in steady state fluid flows: exact and approximate solutions for propagating MHD modes.
}

\author{
Filippo Pantellini \\ and \\ Léa Griton \\ LESIA, Observatoire de Paris, PSL Research University, CNRS, Sorbonne Universités, UPMC \\ Univ. Paris 06, Univ. Paris Diderot, Sorbonne Paris Cité
}

\begin{abstract}
The spatial structure of a steady state plasma flow is shaped by the standing modes with local phase velocity exactly opposite to the flow velocity. The general procedure of finding the wave vectors of all possible standing MHD modes in any given point of a stationary flow requires numerically solving an algebraic equation. We present the graphical procedure (already mentioned by some authors in the 1960's) along with the exact solution for the Alfvén mode and approximate analytic solutions for both fast and slow modes. The technique can be used to identify MHD modes in space and laboratory plasmas as well as in numerical simulations.
\end{abstract}

Subject headings: Magnetohydrodynamics (MHD), Waves, planets and satellites: magnetic fields, methods: analytical

\section{Introduction}

For a characteristic plasma mode propagating at phase velocity $u_{\phi}\left(\theta_{k B}\right)$, where $\theta_{k B}$ is the angle between the wave vector $\vec{k}$ and the local magnetic field $\vec{B}$, standing planar fronts may be supported in a plasma flowing at a given velocity $\vec{u}_{f}$. However, standing fronts can only exist under specific circumstances. For example, in the case of ordinary sound waves for which the phase velocity is $\pm c$, independently of direction, standing modes can only exist if the flow velocity satisfies the condition $u_{f}=c$. For strongly angle dependent phase velocities, however, standing fronts can exist under much less restrictive conditions. Let us consider the case of a mode with phase velocity $u_{\phi}=\omega / k$ in the plasma rest frame ( $\omega$ is the angular frequency and $k$ the wave vector magnitude of the mode). Let us further assume that the mode phase velocity only depends on its propagation angle $\theta_{k B}$ with respect to the local magnetic field $\vec{B}$, i.e.

$$
u_{\phi}=u_{\phi}\left(\theta_{k B}\right)
$$


The Alfvén mode with a phase speed

$$
u_{\phi A}^{2}=a^{2} \cos ^{2} \theta_{k B}
$$

and the fast and slow modes with phase speeds:

$$
u_{\phi S, F}^{2}=\frac{1}{2}\left\{1+a^{2} \pm \sqrt{\left(1+a^{2}\right)^{2}-4 a^{2} \cos ^{2} \theta_{k B}}\right\}
$$

are examples of the generic expression (1). In (3) the sign "-" ("+") corresponds to the slow (fast) mode. Also, note that in the above equations and throughout the whole paper velocities have been normalized to the adiabatic sound speed $c$. In these velocity units $a=c_{A} / c=\sqrt{2}(\beta \gamma)^{-1 / 2}$ is the normalized Alfvén speed where $\gamma$ is the adiabatic index and $\beta$ the local thermal to magnetic pressure ratio of the plasma.

An illustration of the graphical procedure to identify standing modes in a flowing fluid (e.g. Spreiter and Alksne 1970) is shown in Fig. 1. The graphical procedure is based on Thales' theorem which states that a circle inscribed triangle with one of its sides being equal to the diameter of the circle is right-angled. Thus, the two triangles defined by the pairs of vectors $\left(\vec{u}_{f}, \vec{u}_{1}\right)$ and $\left(\vec{u}_{f}, \vec{u}_{2}\right)$ in Fig. 1 are by construction right-angled. The tips of both $\vec{u}_{1}$ and $\vec{u}_{2}$ being located on the phase velocity curve $u_{\phi}$, the two vectors do also represent the phase velocity of two modes (the dotted lines showing the associated front) for which the propagation velocity is equal and opposite to the flow velocity component normal to the fronts.

We note that $\vec{u}_{1}$ and $\vec{u}_{2}$ are merely particular solutions confined to plane defined by the vectors $\vec{B}$ and $\vec{u}_{f}$ (the $(x, z)$ plane). The general solution requires computing the intersection of a sphere with the rotationally invariant surface defined by either (2) or (3). The sphere is the one obtained by rotating the dashed circle of Fig 1 about the axis defined by the velocity vector $\vec{u}_{f}$.

In this paper, we do stick to the reference frame of Fig. 1 where the expressions for the phase velocities of the MHD modes are particularly simple. However, this frame is not the most practical one in most applications as both the magnetic field and the flow velocity orientations are generally a function of position. The construction of the matrix $M$ which transforms the vector components from an arbitrary frame of reference (where the flow is stationary) into the frame of Fig. 1 is described in Appendix A.

\section{General solution}

Basically, the problem of finding the standing modes in a flowing plasma reduces to the problem of finding the intersection in velocity space of the surface of a sphere of radius $u_{f} / 2$ passing through the origin $O$ and the surface of revolution defined by the phase velocity $u_{\phi}^{2}\left(\theta_{k B}\right)$. Let us assume (with no loss of generality) that the flow velocity vector is in the $\left(u_{x}, u_{z}\right)$ plane so that the equation of the sphere is

$$
\left(u_{x}-u_{x 0}\right)^{2}+u_{y}^{2}+\left(u_{z}-u_{z 0}\right)^{2}=\left(\frac{1}{2} u_{f}\right)^{2}
$$




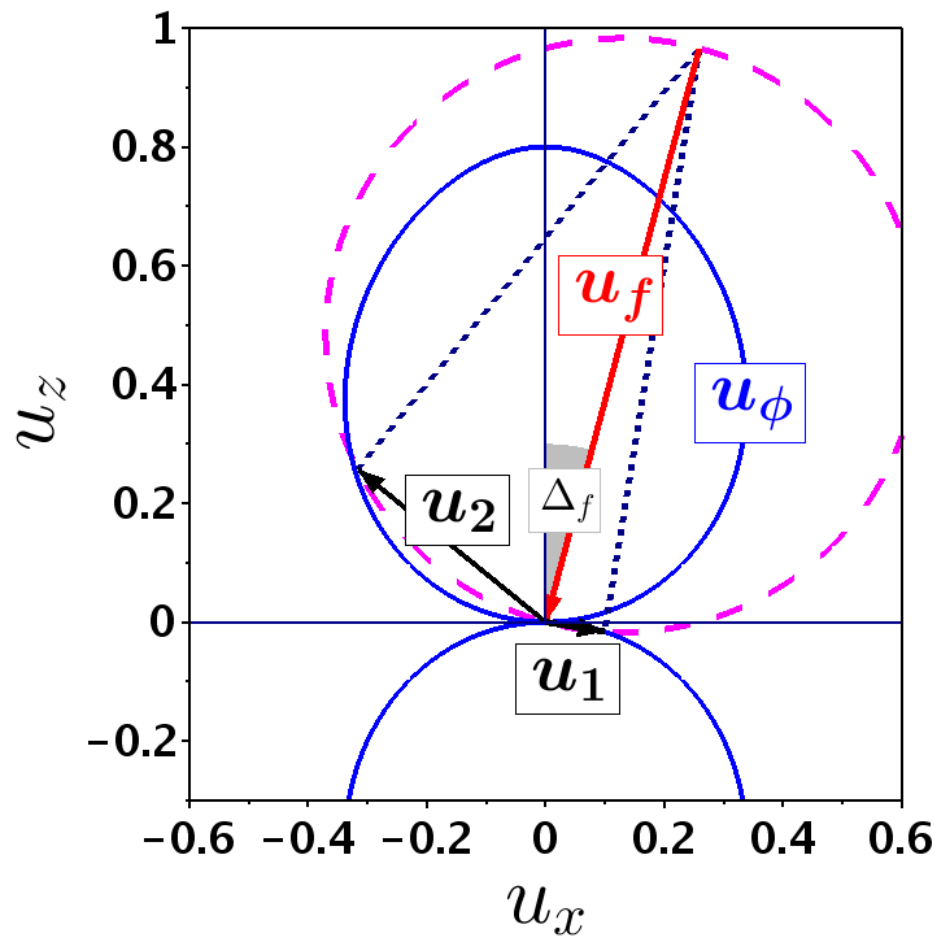

Fig. 1.- Graphical procedure to identify standing modes in a plasma flowing at velocity $\vec{u}_{f}$. In the figure, $u_{\phi}$ represents the phase velocity of the selected mode in the plasma frame of reference. The magnetic field points along the $z$ axis and the angle $\theta_{k B}$ in equation (3) represents the angle with respect to $z$. The orientation of the $z$ axis is such that the $z$ component of the flow speed $\vec{u}_{f}$ (measured in the frame where the flow is stationary) is zero or negative. The $x$ axis is such that $\vec{u}_{f}$ has negative $x$ and no $y$ component. The $B_{z}$ component of the magnetic field may be positive or negative. The two vectors $\vec{u}_{1}$ and $\vec{u}_{2}$ (given by the intersection of a circle defined by $\vec{u}_{f}$ and the phase velocity $u_{\phi}$ ) are the phase velocities and propagation direction (in the plasma frame) of the two standing slow mode fronts with wave vectors in the $(x, z)$ plane. $\Delta_{f}$ is the angle between the $z$ axis and $\vec{u}_{f}$.

Denoting $\Delta_{f}$ as the angle between the flow velocity $\vec{u}_{f}$ and the $z$ axis (cf Fig 1 ), it follows that the velocity components of the center of the sphere are given by

$$
\begin{aligned}
& u_{x 0}=\frac{1}{2} u_{f} \sin \Delta_{f} \\
& u_{z 0}=\frac{1}{2} u_{f} \cos \Delta_{f}
\end{aligned}
$$

where the $z$ axis is oriented such to have $0<\Delta_{f} \leq \pi / 2$, meaning that the magnetic field can be either parallel or anti-parallel with respect to the $z$ axis. Setting $u^{2}=u_{x}^{2}+u_{y}^{2}+u_{z}^{2}$ the squared velocity with respect to the origin, we write the velocity components of an arbitrary point in velocity 
space as

$$
\begin{aligned}
& u_{x}=|u| \sin \theta \cos \varphi \\
& u_{y}=|u| \sin \theta \sin \varphi \\
& u_{z}=|u| \cos \theta
\end{aligned}
$$

where $0<\theta<\pi$ is the polar angle with respect to the $z$ axis and $0 \leq \varphi<2 \pi$ is the azimuthal angle with respect to the $x$ axis. One can then describe the surface of the sphere given by (4) in the more explicit form:

$$
|u|=u_{f}\left\{\sin \Delta_{f} \sin \theta \cos \varphi+\cos \Delta_{f} \cos \theta\right\} .
$$

The solution of the problem consists in searching the family of modes for which the phase velocity $\left|u_{\phi}(\theta)\right|$ along the direction $\theta$ is located on the spherical surface defined by (10), i.e.

$$
\left|u_{\phi}(\theta)\right|=u_{f}\left\{\sin \Delta_{f} \sin \theta \cos \varphi+\cos \Delta_{f} \cos \theta\right\} .
$$

The potentially difficult step is the determination of the propagating direction $\theta=\theta\left(\Delta_{f}, u_{f}, a, \varphi\right)$ defined by (11). Once the propagation direction $\theta(\varphi)$ of the standing mode is known, the module of the associated phase velocity is trivially given by the phase velocity $u_{\phi}(\theta)$ of the corresponding mode given by either (2) or (3).

\section{Solution for the Alfvén mode}

For the Alfvén mode one has to solve (11) with the corresponding mode's phase velocity (2), i.e.

$$
a|\cos \theta|=u_{f}\left\{\sin \Delta_{f} \sin \theta \cos \varphi+\cos \Delta_{f} \cos \theta\right\} .
$$

As illustrated in Fig. 1, two solutions do generally exist on either the upper $(\cos \theta>0)$ or the lower $(\cos \theta<0)$ lobe of the phase velocity surface.

\subsection{Solution $\mathbf{A} 1$ for $\cos \theta<0$ :}

In this case the intersection curve is located on the lower lobe of the phase velocity surface and the left hand side of (12) can be written as $-a \cos \theta$. Solving (12) for $\theta$ leads to

$$
\left.\mathrm{A} 1: \tan \theta_{1}=-\frac{a / u_{f}+\cos \Delta_{f}}{\sin \Delta_{f} \cos \varphi}, \text { with } \varphi \in\right]-\pi / 2, \pi / 2[\text {. }
$$

We note that the condition $\cos \varphi>0$ in (13) is a consequence of $\tan \theta_{1}<0$ (lower lobe). The orientation angle $\theta_{1}$ (corresponding to solution $\vec{u}_{1}$ in Fig. 1) is obtained by setting $\varphi=0$ in (13). 


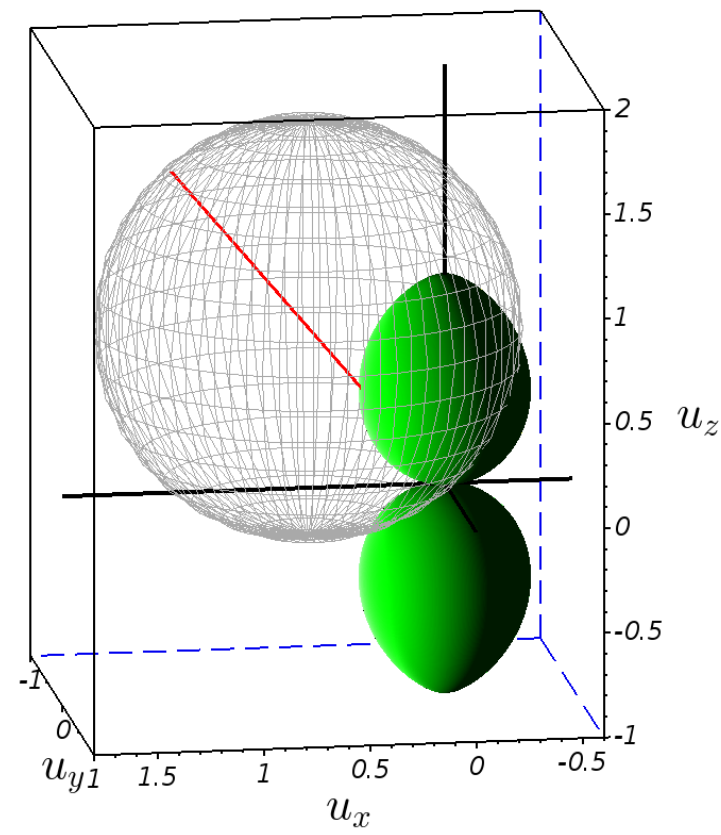

Fig. 2. - Three dimensional view showing both the upper and lower lobes of the phase velocity $u_{\phi S}$ and the sphere of radius $u_{f} / 2$ centered in $-\vec{u}_{f} / 2$. The red line represents the $-\vec{u}_{f}$ direction and the black lines the axes of the coordinate system. Generally, the intersection of the sphere with the $u_{\phi S}$ surface splits into two curves characterized by $u_{z}>0$ and $u_{z}<0$, respectively. These curves represent the phase velocity of all possible modes (in this case slow modes) which are standing in the flow $\vec{u}_{f}$.

\subsection{Solution $\mathbf{A 2}$ for $\cos \theta>0$ :}

In this case the intersection curve is located on the upper lobe of the phase velocity surface but no restrictions on the sign of $\cos \varphi$ can be assumed in this case (see below). The left hand side of (12) can be written as $a \cos \theta$. Again, solving (12) for $\theta$ leads to

$$
\mathrm{A} 2: \tan \theta_{2}=\frac{a / u_{f}-\cos \Delta_{f}}{\sin \Delta_{f} \cos \varphi} .
$$

The requirement $\tan \theta_{2}>0$ implies two distinct cases:

1. $u_{f}>a / \cos \Delta_{f}$ and $\cos \varphi<0$ (i.e. $u_{x}<0$ )

2. $u_{f}<a / \cos \Delta_{f}$ and $\cos \varphi>0$ (i.e. $u_{x}>0$, as for solution A1) 


\section{Approximate solution for the slow mode}

Adopting the general slow mode phase velocity expression (3) with the " - "sign does not allow to produce analytic solutions for the standing modes as in the case of the Alfvén mode. However, in the limit

$$
\frac{4 a^{2}}{\left(1+a^{2}\right)^{2}} \cos ^{2} \theta_{k B} \leq \cos ^{2} \theta_{k B} \ll 1
$$

the slow mode phase velocity can be approximated as

$$
u_{\phi S}^{2} \simeq A^{2} \cos ^{2} \theta_{k B}, \text { where } A^{2} \equiv \frac{a^{2}}{1+a^{2}} .
$$

This is the same dispersion relation as for the Alfvén mode with the velocity $A$ replacing the Alfvén velocity $a$. The standing modes solutions for the Alfvén mode do therefore work for the slow mode as well as long as its phase velocity can be approximated by an expression of the form (16). The dotted curves in Fig. 3 illustrate the quality of the approximation in the less favorable domain $a \sim 1$.
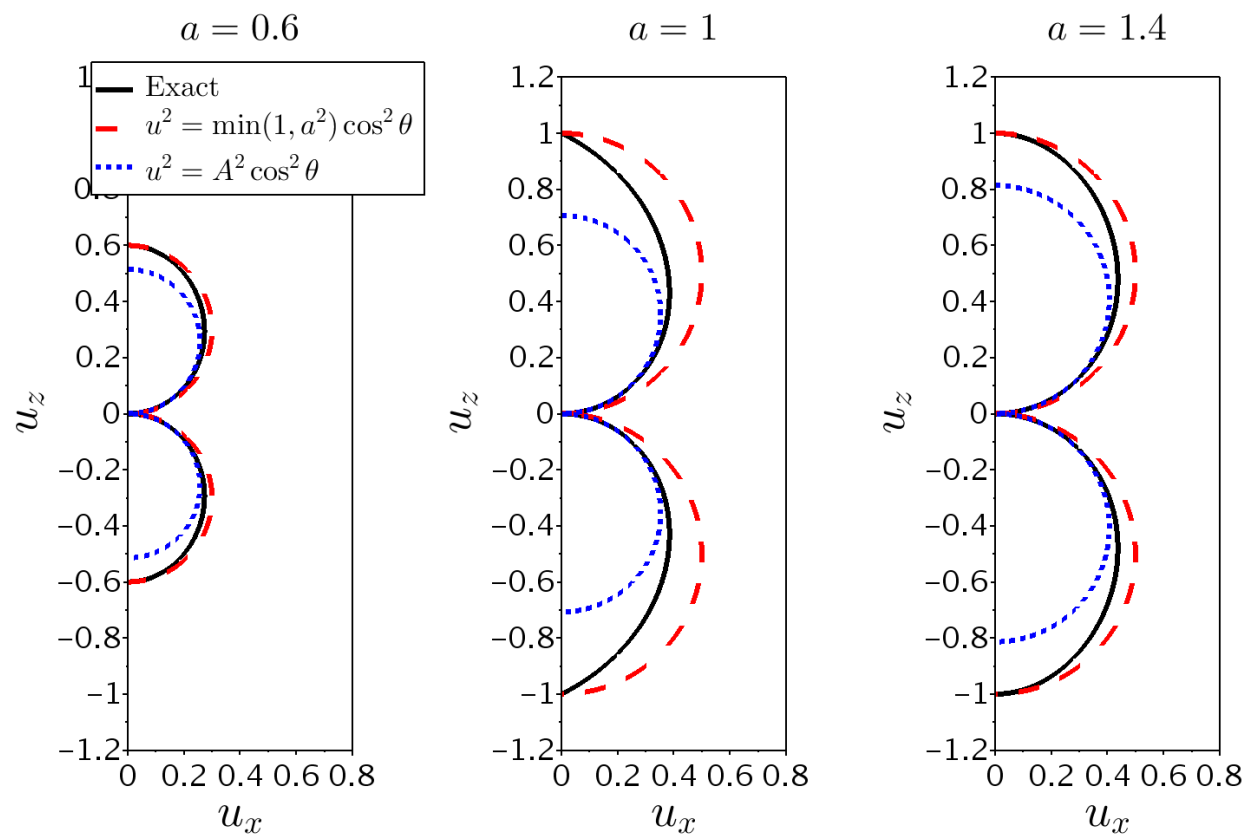

Fig. 3.- Approximations of the slow mode phase velocity for three different values of the Alfvén velocity $a$. The two approximations do asymptotically approach the exact solution for either $a \rightarrow 0$ or $a \rightarrow \infty$.

Using the approximated dispersion for the slow mode (16) the solutions S1 and S2 are trivial transcriptions of the A1 and A2 solutions for the Alfvén mode:

$$
\left.\mathrm{S} 1: \tan \theta_{1}=-\frac{A / u_{f}+\cos \Delta_{f}}{\sin \Delta_{f} \cos \varphi}, \text { with } \varphi \in\right]-\pi / 2, \pi / 2[
$$


and

$$
\text { S2: } \tan \theta_{2}=\frac{A / u_{f}-\cos \Delta_{f}}{\sin \Delta_{f} \cos \varphi} \text {. }
$$

As for the Alfvén solution A2, the slow mode solution S2 with the requirement $\tan \theta_{2}>0$ admits two cases:

1. $u_{f}>A / \cos \Delta_{f}$ and $\cos \varphi<0$ (i.e. $u_{x}<0$ )

2. $u_{f}<A / \cos \Delta_{f}$ and $\cos \varphi>0$ (i.e. $u_{x}>0$, as for solution $\mathrm{S} 1$ ).

A representative example illustrating the shape of both S1 and S2 curves in velocity space is shown in Fig. 4.

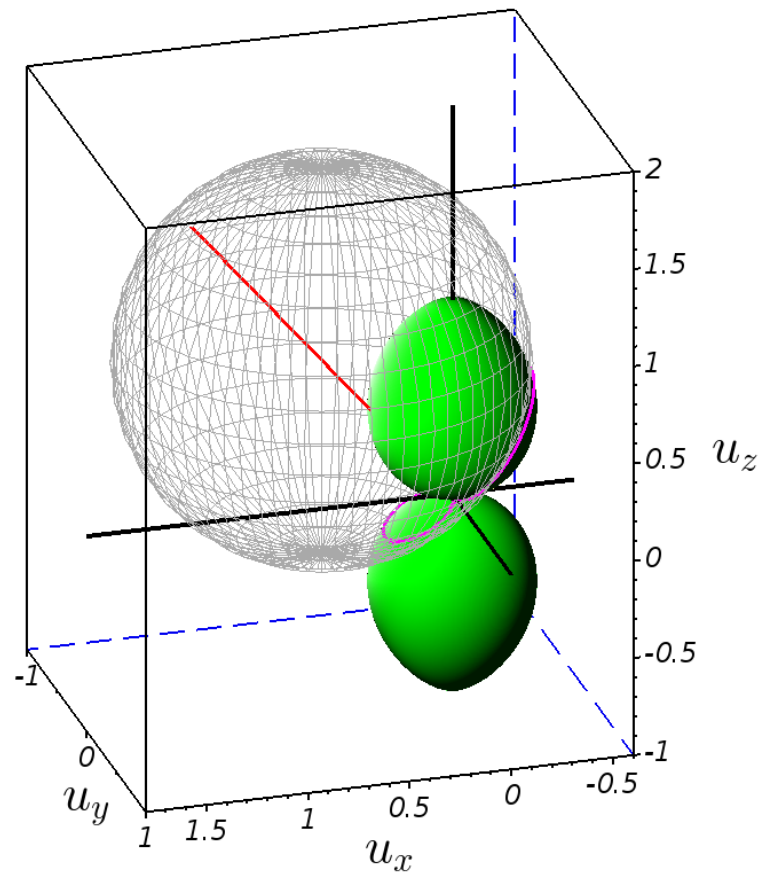

Fig. 4.- Figure format is the same as in Fig. 2. In this particular example, the approximate curves S1 and S2 from (17) and (18) do closely follow the exact solutions defined by the intersection of the flow velocity sphere (gridded sphere) with the two lobes of the slow mode phase velocity surface.

We conclude this section by noting that a different approximation than (16) can be obtained by assuming $a \gg 1$ and $a \ll 1$. The resulting slow mode phase velocity has the same $\theta_{k B}$ dependence as (16) with the coefficient $A^{2}$ replaced by $\min \left(1, a^{2}\right)$. Both approximations do an excellent job for $a \gg 1$ and $a \ll 1$. For $a \simeq 1$, the two approximations are mediocre, the one given by (16) having the advantage of doing well (by construction) for $\cos ^{2} \theta_{k B} \ll 1$. 
In the next section, we show contour plots of the error of S1 and S2 resulting from the use of the slow mode phase speed approximation (16) for a close to unity where the phase speed approximation (16) is poorest.

\subsection{Error of the approximation for the slow mode}

Fig. 5 shows the difference $\delta \theta \equiv \theta_{\text {ex }}-\theta_{\text {app }}$ between the approximate solution $\theta_{\text {app }}$ (from equations (17) and (18)) and the exact solution $\theta_{\text {ex }}$ obtained by numerically solving (11) with the exact expression of the slow mode phase velocity (3). Error profiles have been computed for the two standing modes in the $(x, z)$ plane (which can take values $\varphi=0$ for S1 and either $\varphi=0$ or $\varphi=\pi$ for S2). The $(x, z)$ plane is the plane where the errors are largest.
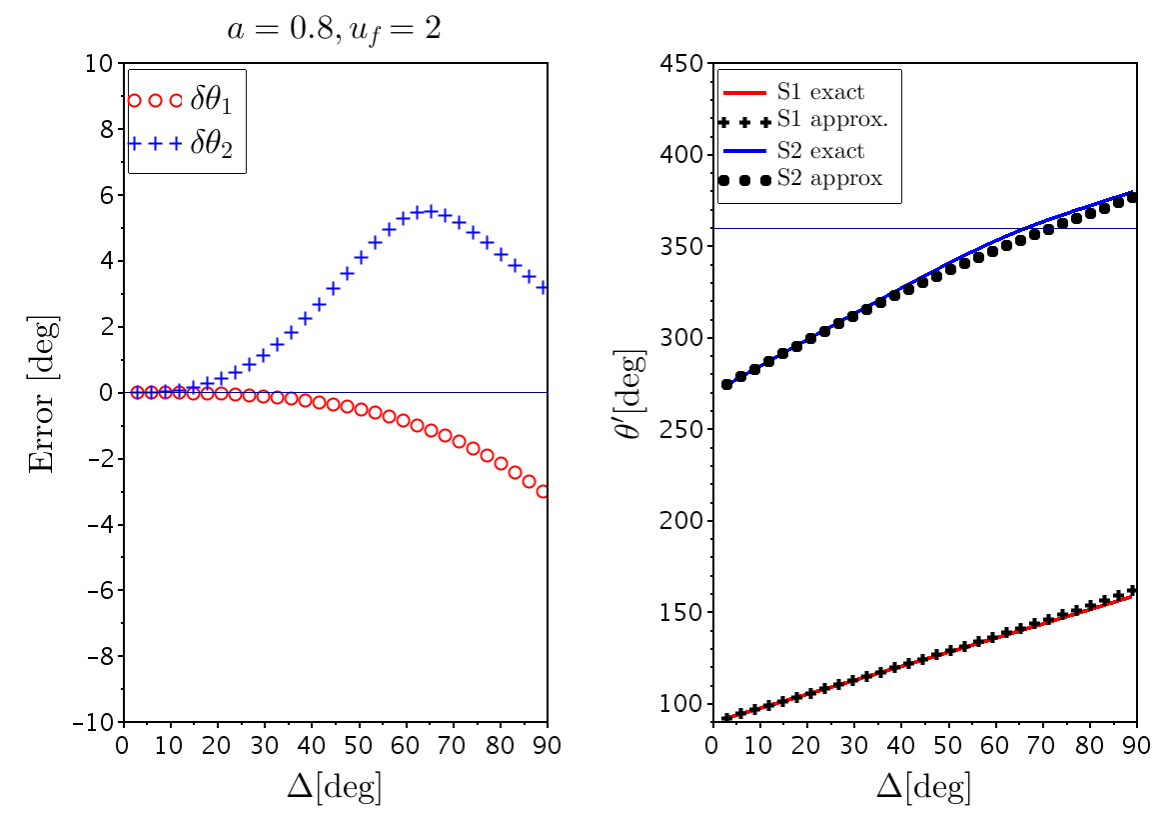

Fig. 5.- Slow mode: Error on the determination of the orientation of the standing fronts for a singular choice of the Alfvén velocity $a$ and the fluid velocity $u_{f}$. Left panel: error $\delta \theta$ for the orientation of the standing slow mode solutions S1 and S2 as a function of the angle $\Delta_{f}$ in the $(x, z)$ plane. As expected the approximation is excellent for small values of $\Delta$. Right panel: approximate and exact orientations of the standing modes. Note that in order to distinguish between the two possible orientations $\varphi=0$ and $\varphi=\pi$ we have introduced the angle $\theta^{\prime}=\theta \cos \varphi /|\cos \varphi|$ which runs from 0 to $2 \pi$ (while $\theta \in[0, \pi]$ ).

By construction the error vanishes for $\Delta_{f} \rightarrow 0\left(\vec{u}_{f}\right.$ and $\vec{B}$ aligned) as in that case the standing mode solutions satisfy $|\cos \theta| \propto \Delta_{f} \rightarrow 0$ which is precisely the limit for the approximate dispersion (16) to asymptotically approach the full dispersion (3). In general the error for S2 is larger than for S1. This is due to the fact that curve S2, which runs over the upper lobe of the phase velocity 
surface (see Fig. 4 and also Fig. 1) reaches higher $u_{z}$, i.e. larger $|\cos \theta|$ values (and thus lower precision) than $\mathrm{S} 1$.

Slow mode, error for $\theta_{1}(a=0.8)$
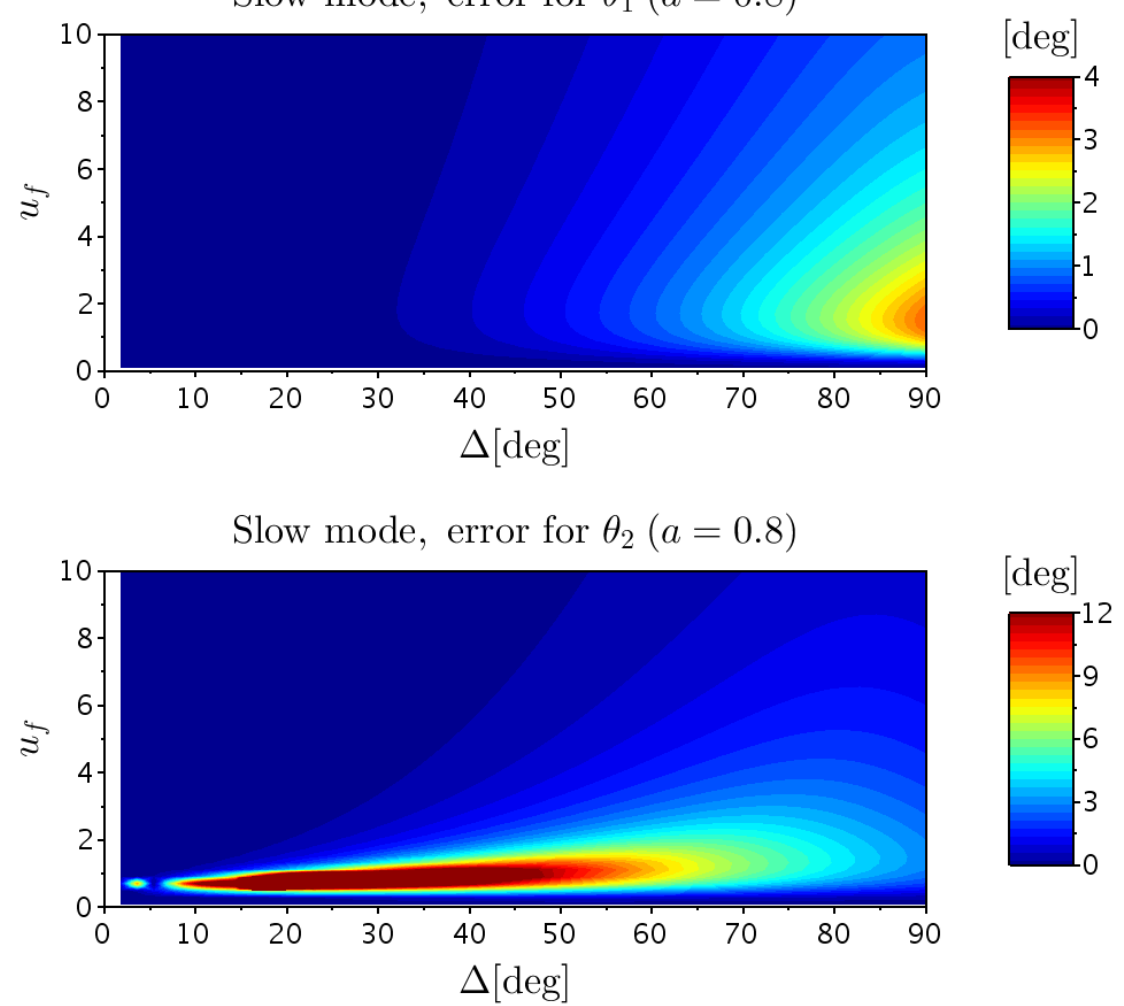

Fig. 6.- Absolute error $|\delta \theta|$ for the orientation of the standing fronts $\theta_{1}$ and $\theta_{2}$ for solutions S1 (17) and S2 (18), respectively. Note the difference in scale used for the two plots. As in Fig. 5 the error has been computed for the $(x, z)$ plane where the largest errors occur.

An overview of the absolute error for S1 and S2 as a function of both $\Delta_{f}$ and $u_{f}$ is shown in Fig. 6. The difference between the two plots is striking. As already noted in Fig. 5 the error is generally larger for solution $\mathrm{S} 2$. Indeed, while the error $\left|\delta \theta_{1}\right|$ is less than $3^{\circ}$ over the whole domain, $\left|\delta \theta_{2}\right|$ exceeds $12^{\circ}$ for $u_{f} \lesssim 1$ over an extended range of $\Delta_{f}$ angles. Thus, while $\mathrm{S} 1$ does generally provide a rather accurate approximation, S2 must be used with care.

The error being due in part to the $\cos ^{2} \theta \ll 1$ restriction of the phase velocity approximation (16), one may be tempted to discard solutions S1 and S2 corresponding to $\cos ^{2} \theta \gtrsim 1 / 2$. However, error may remain reasonably small for essentially all accessible $\theta$ values, even for $a \sim 1$ (the worst possible regime), as illustrated in the right panel of Fig. 5 . 


\section{Approximate solutions for the fast mode}

Approximate analytic solutions for the standing fast modes can be obtained following the same procedure as for the slow mode. However, given the form of the fast mode phase velocity

$$
u_{\phi F}^{2}\left(\theta_{k B}\right)=\frac{1}{2}\left\{1+a^{2}+\sqrt{\left(1+a^{2}\right)^{2}-4 a^{2} \cos ^{2} \theta_{k B}}\right\}
$$

a first order development limited to small values of $\left|\cos ^{2} \theta_{k B}\right| \ll 1$ is not the best choice. A more general approximation can be obtained by taking the limits $a \gg 1$ and $a \ll 1$, respectively. For $a \gg 1,(19)$ reduces to

$$
\begin{aligned}
u_{\phi F}^{2}\left(\theta_{k B}\right) & \simeq \frac{1}{2}\left\{1+a^{2}+\left(1+a^{2}\right)\left(1-\frac{2 \cos ^{2} \theta_{k B}}{a^{2}}\right)\right\} \\
& \simeq 1+a^{2}-\cos ^{2} \theta_{k B} .
\end{aligned}
$$

Likewise, for $a \ll 1$ one has:

$$
\begin{aligned}
u_{\phi F}^{2}\left(\theta_{k B}\right) & \simeq \frac{1}{2}\left\{1+a^{2}+\left(1+a^{2}\right)\left(1-2 a^{2} \cos ^{2} \theta_{k B}\right)\right\} \\
& \simeq 1+a^{2}-a^{2} \cos ^{2} \theta_{k B} .
\end{aligned}
$$

The two above expressions can be merged into a single one:

$$
u_{\phi F}^{2}\left(\theta_{k B}\right)=1+a^{2}-\min \left(1, a^{2}\right) \cos ^{2} \theta
$$

The quality of the approximation (24) can be appreciated in Fig. 7 for three values of $a$. Introducing
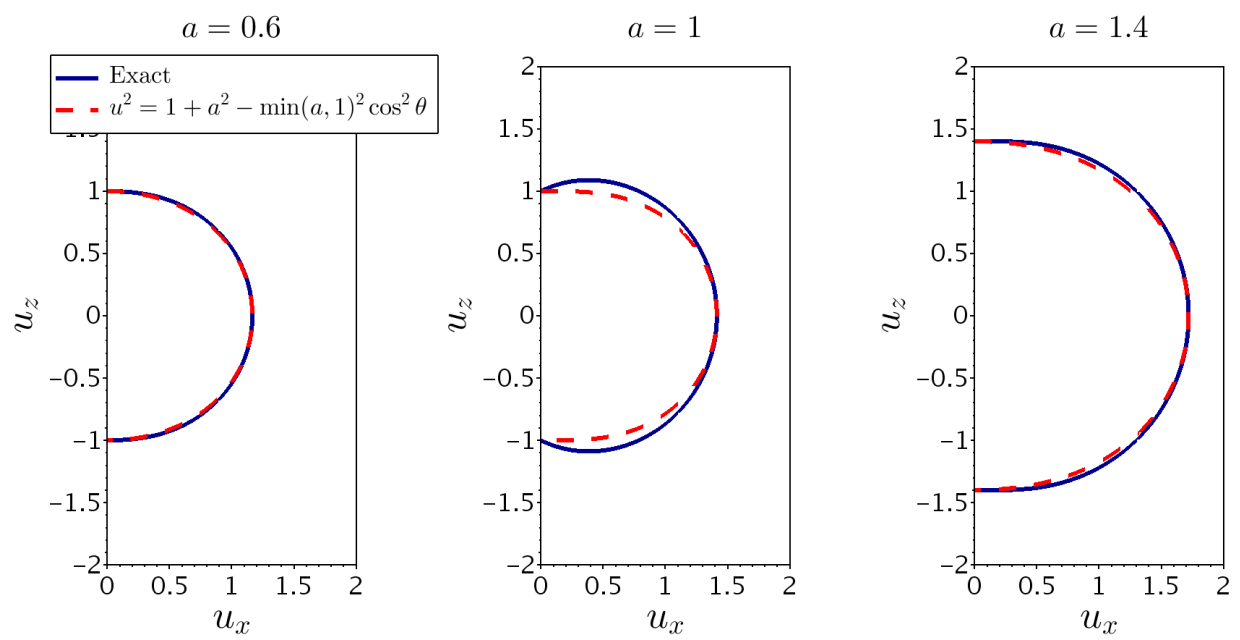

Fig. 7.- Approximation of the fast mode velocity for different values of the normalized Alfvén velocity $a$. It is worth noting that the approximation is best for $a \ll 1$ or $a \gg 1$. For small angles, the solution is exact regardless of the value of $a$. 
$B^{2} \equiv \min \left(1, a^{2}\right), C^{2} \equiv 1+a^{2}$, and replacing the approximate expression (24) into the squared version of (11) leads to

$$
C^{2}-B^{2} \cos ^{2} \theta=u_{f}^{2}\left\{\sin \Delta_{f} \sin \theta \cos \varphi+\cos \Delta_{f} \cos \theta\right\}^{2}
$$

Further operations and simplifications allow to express the relation as a quadratic function of $\tan \theta$ :

$$
\lambda_{1} \tan ^{2} \theta+\lambda_{2} \tan \theta+\lambda_{3}=0
$$

where

$$
\begin{aligned}
& \lambda_{1} \equiv \sin ^{2} \Delta_{f} \cos ^{2} \varphi-\frac{C^{2}}{u_{f}^{2}} \\
& \lambda_{2} \equiv \sin \left(2 \Delta_{f}\right) \cos \varphi \\
& \lambda_{3} \equiv \cos ^{2} \Delta_{f}+\frac{B^{2}-C^{2}}{u_{f}^{2}} .
\end{aligned}
$$

The two solutions of (26) are

$$
\tan \theta_{1,2}=-\frac{\sin \left(2 \Delta_{f}\right) \cos \varphi \pm \sqrt{D}}{2\left(\sin ^{2} \Delta_{f} \cos ^{2} \varphi-\frac{C^{2}}{u_{f}^{2}}\right)}
$$

where $\theta_{1}$ and $\theta_{2}$ correspond to the sign "+ " and "- ", respectively. Real solutions of (30) require $D \equiv \lambda_{2}^{2}-4 \lambda_{1} \lambda_{3} \geq 0$, i.e.

$$
D=\frac{4 C^{2}\left(C^{2}-B^{2}\right)}{u_{f}^{2}}\left(\frac{\sin ^{2} \Delta_{f} \cos ^{2} \varphi}{C^{2}}+\frac{\cos ^{2} \Delta_{f}}{C^{2}-B^{2}}-\frac{1}{u_{f}^{2}}\right) \geq 0
$$

complemented by the additional requirement (resulting from (11))

$$
\sin \Delta_{f} \sin \theta_{1,2} \cos \varphi+\cos \Delta_{f} \cos \theta_{1,2} \geq 0 .
$$

The special case $\Delta_{f}=0$ thus implies $0 \leq \theta_{1,2} \leq \pi / 2$. Otherwise, one may also want to write (32) in terms of a condition on $\varphi$ :

$$
\cos \varphi \geq \frac{-1}{\tan \Delta_{f} \tan \theta_{1,2}}=\frac{2}{\tan \Delta_{f}} \frac{\sin ^{2} \Delta_{f} \cos ^{2} \varphi-\frac{C^{2}}{u_{f}^{2}}}{\sin \left(2 \Delta_{f}\right) \cos \varphi \pm \sqrt{D}} .
$$

The reason for imposing (32) stems from the fact that (25), which has been solved to obtain (30), allows both negative and positive values for the left hand side of (32) in clear contradiction with (11). A characteristic example showing the loci of the standing fast modes using the approximate expression (30) is shown in Fig. 8. It should be noted that the simple closed curve resulting from 


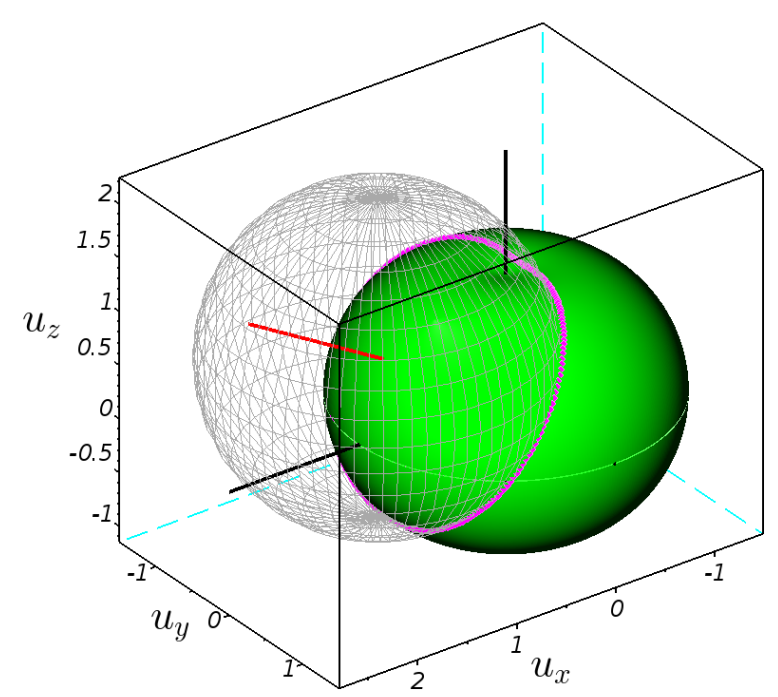

Fig. 8. - Fast mode. Unlike the Alfvén or the slow mode, the fast mode phase velocity surface does not have two lobes. Accordingly, its intersection with the sphere of diameter $u_{f}$ (centered in $-\vec{u}_{f}$ ) makes just one simple closed curve instead of two closed curves joining at the origin.

the intersection of the $-\vec{u}_{f} / 2$ centered sphere and the fast mode phase velocity surface generally mixes the two solutions $\theta_{1}$ and $\theta_{2}$.

The 3D solution represents a single curve, which is constituted, depending on the value of $\Delta_{f}$, by either one of the solutions or by a combination of both. It is important to note that in many cases no standing fast mode solution exist. Graphically, this corresponds to the case of the $u_{f}$ sphere being completely contained inside the dispersion relation surface or, in the approximate solution (30), to the condition $D<0$.

We note indeed that the constraint $D \geq 0$ for real solutions to exist implies the flow velocity to exceed a limiting value

$$
u_{f}^{2} \geq\left(\frac{\cos ^{2} \Delta_{f}}{\max \left(1, \mathrm{a}^{2}\right)}+\frac{\sin ^{2} \Delta_{f}}{C^{2}} \cos ^{2} \varphi\right)^{-1}
$$

\subsection{Error of the approximation for the fast mode}

An example of $\Delta$ dependence of the errors for both $\mathrm{F}_{1,2}$ solutions (corresponding to the two solutions $\theta_{1,2}$ from (30)) is shown in Fig. 9. The figure shows that the error associated with this approximation is generally $<1^{\circ}$, except near the lower limit of $u_{f}$ and values of $a$ close to unity as illustrated in Fig. 10 for the particular case $a=1.1$.

As already pointed out, the approximate expression for the fast mode's phase velocity (24) 

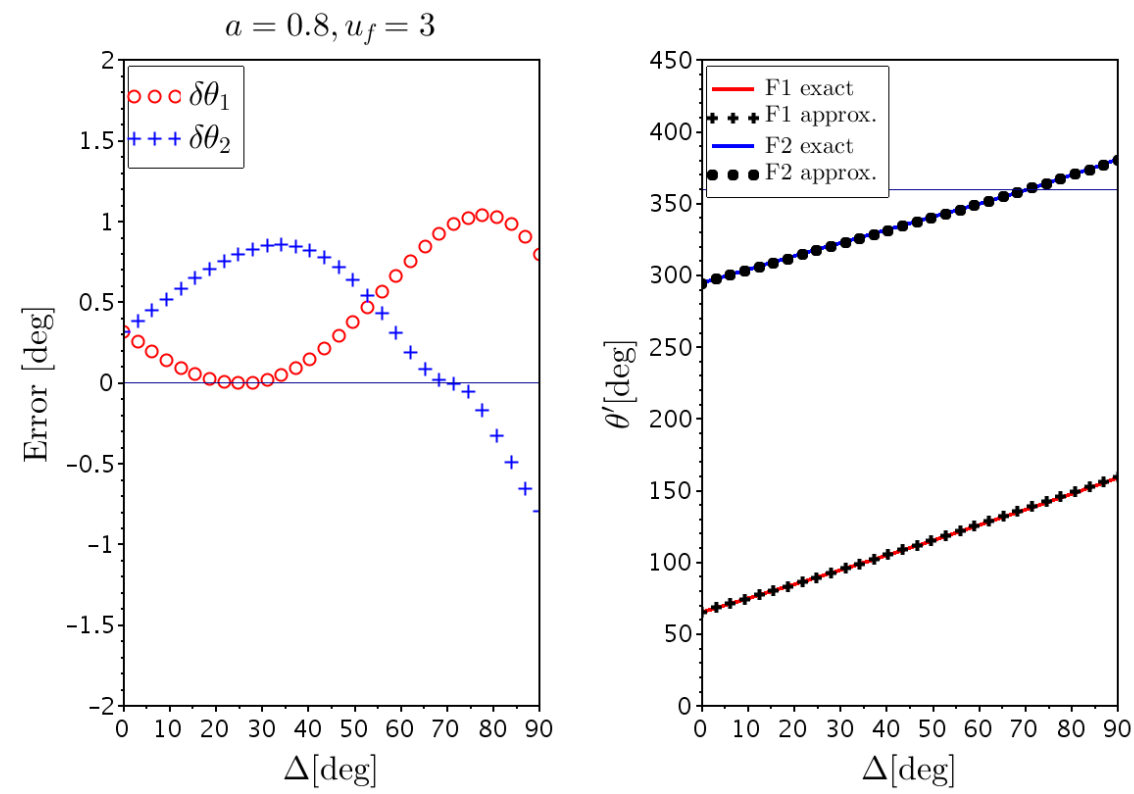

Fig. 9.- Fast mode: Error on the determination of the orientation of the standing fronts for a singular choice of the Alfvén velocity $a$ and the fluid velocity $u_{f}$. Format is the same as for Fig. 5.

becomes increasingly accurate as the Alfvén velocity $a$ departs from unity meaning that $\mathrm{F}_{1,2}$ are exact solutions for $a \rightarrow 0, \infty$. The contour plots in Fig. 11 do indeed show that already for moderate departures of $a$ from unity, the error is significantly smaller over most of the $\left(\Delta, u_{f}\right)$ parameter space and large errors concentrated in a increasingly narrow region near the lower limit for $u_{f}$.

\section{Sample applications}

In this section, we discuss a few case examples in order to illustrate and test the validity of the above described method to identify standing MHD modes in a stationary plasma flow. As previously explained, for given plasma parameters, flow and magnetic field orientations, an infinite number of standing modes are generally possible. As shown in Figures 4 and 8, the tips of all possible standing phase velocity vectors describe a closed curve in the three-dimensional velocity space. In general, only a subset of all possible standing modes is effectively compatible with the imposed boundary conditions. In numerical simulations, laboratory or natural plasmas, it is often possible to identify the orientation of the dominant wave vector by measuring the gradient of some local quantity such as density, pressure, magnetic field etc.. When more than one standing mode is allowed in the direction specified by the gradient, one may use transport ratios to refine the identification process. Transport ratios are dimensionless numbers obtained by the mutual division of two fluctuating quantities in a linear wave. In multi-species Vlasov plasmas, it is generally necessary to compute the transport ratios numerically (e.g. Lacombe et al. 1992). In the MHD 

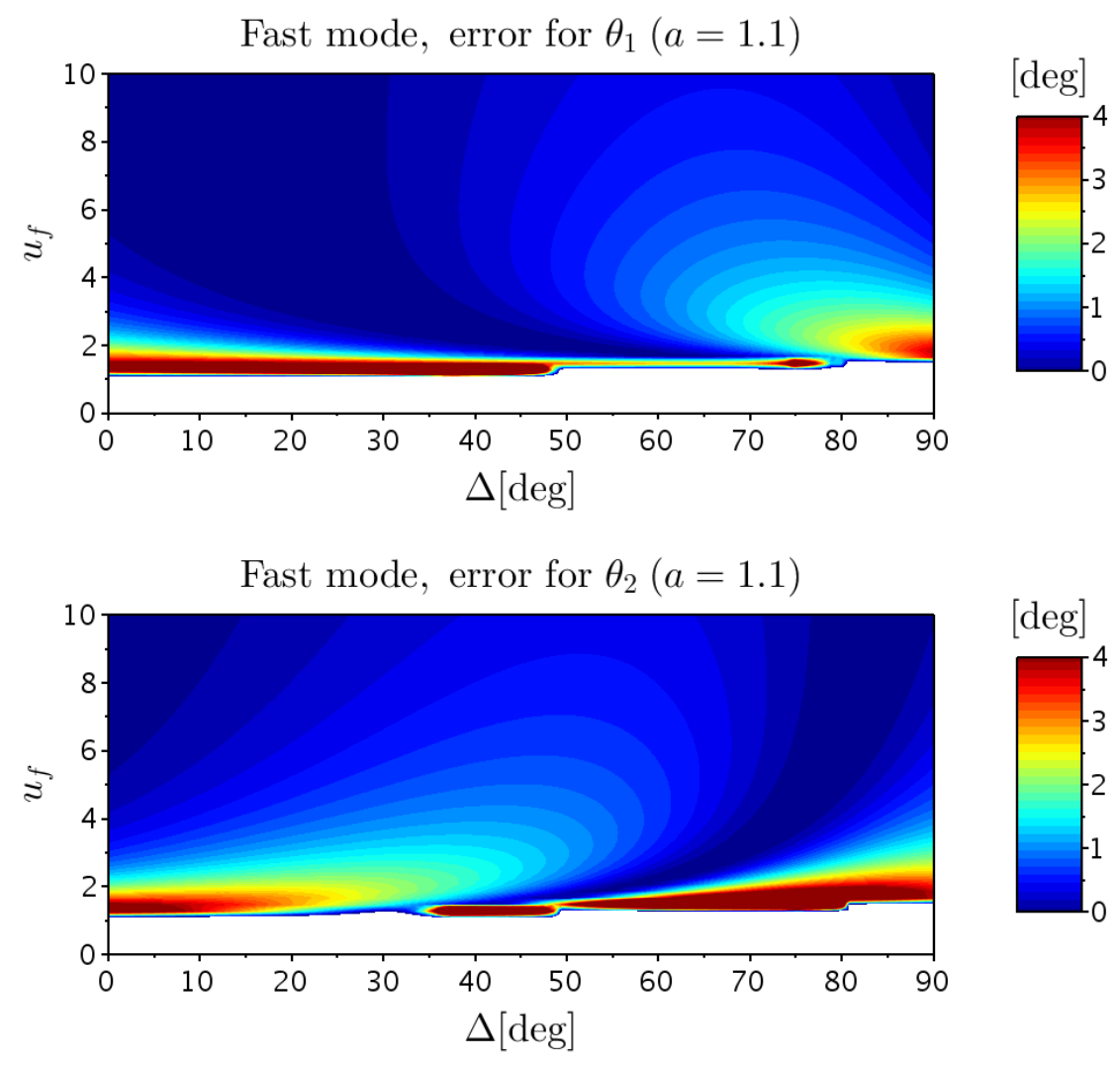

Fig. 10.- Absolute errors for the two standing fast modes $(30)$ in the $(x, z)$ plane. The regime $a \simeq 1$ is potentially the one with the largest errors, as the approximate expression (24) applies specifically to $a \ll 1$ or $a \gg 1$. No standing fast mode solutions exist in the white shaded regions where condition (34) is not satisfied.

limit, the only two relevant transport ratios are the parallel compressibility $C_{\|}$and the Alfvén ratio $R_{A}$. They are briefly described in Appendix B. The parallel compressibility, which does compare density and magnetic field fluctuations, is the most useful to disentangle fast and slow modes as it is always positive for the former (density and magnetic field vary in phase) and always negative for the latter (density and magnetic field vary in anti-phase). The Alfvén mode being incompressible (no density fluctuation), the associated parallel compressibility is vanishing small. If a doubt subsists, the Alfvén ratio which compares velocity and magnetic fluctuations may be used. Example profiles of the two just mentioned transport ratios as a function of propagation angle $\theta_{k B}$ are shown in Fig 16. 

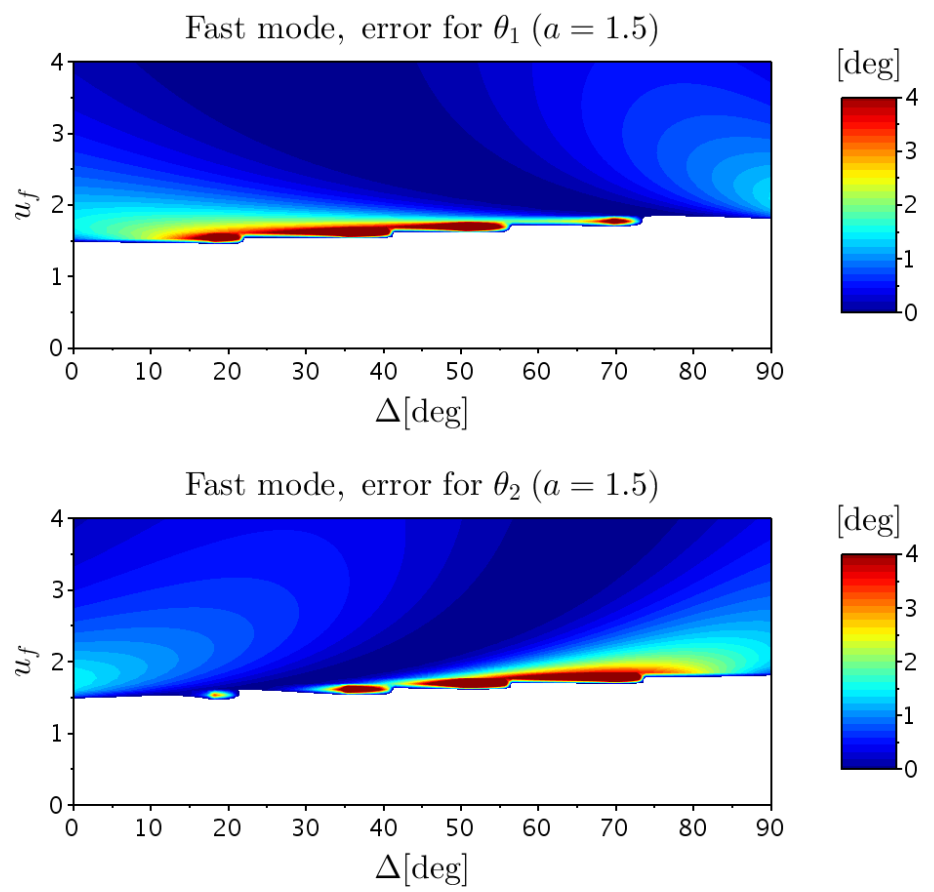

Fast mode, error for $\theta_{1}(a=0.5)$
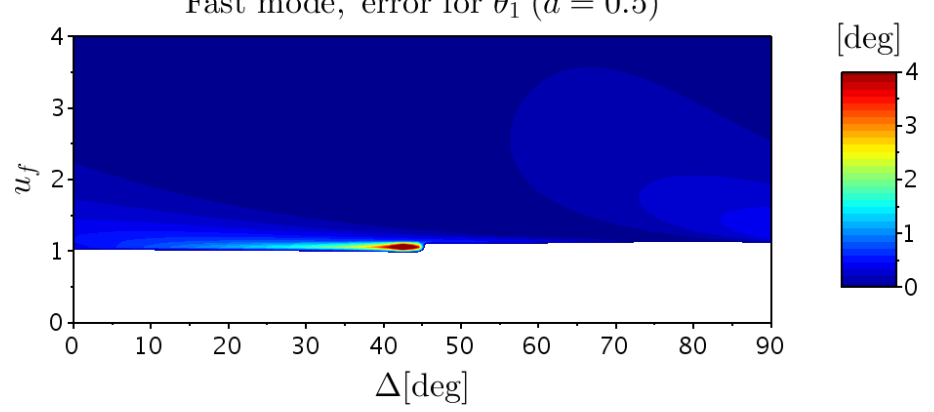

Fast mode, error for $\theta_{2}(a=0.5)$

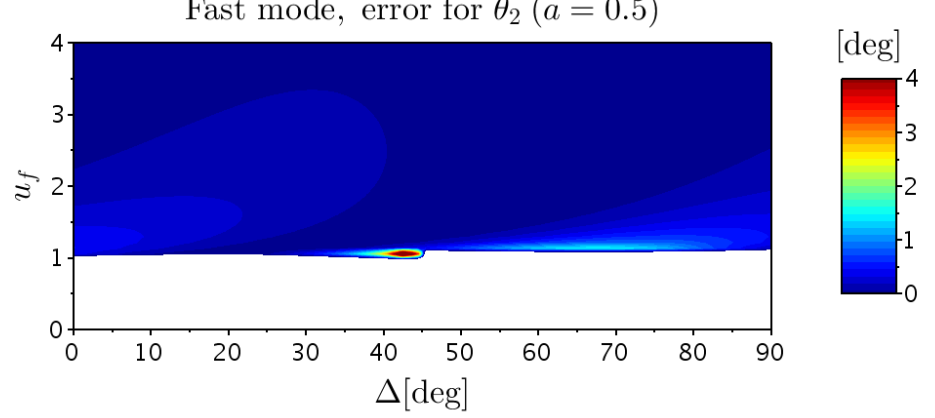

Fig. 11. - Fast mode: Absolute error for the orientation of standing fast mode solutions (30) for $a$ values larger and smaller than unity.

\subsection{Sample application for the fast mode}

Standing fast mode fronts are very common in astrophysical plasmas. The interaction of the solar wind with any of the magnetized planets of the solar system produces such fronts in the form 
of a bow shock. Figure 12 shows a plane cut through a three-dimensional simulation of Mercury's magnetosphere (parameters are those of Pantellini et al. (2015)) with the solar wind flow streaming from left to right. Simulation parameters are such that the solar wind flow, the solar wind magnetic field, and the planet's magnetic axis are in the plane shown in Figure 12.

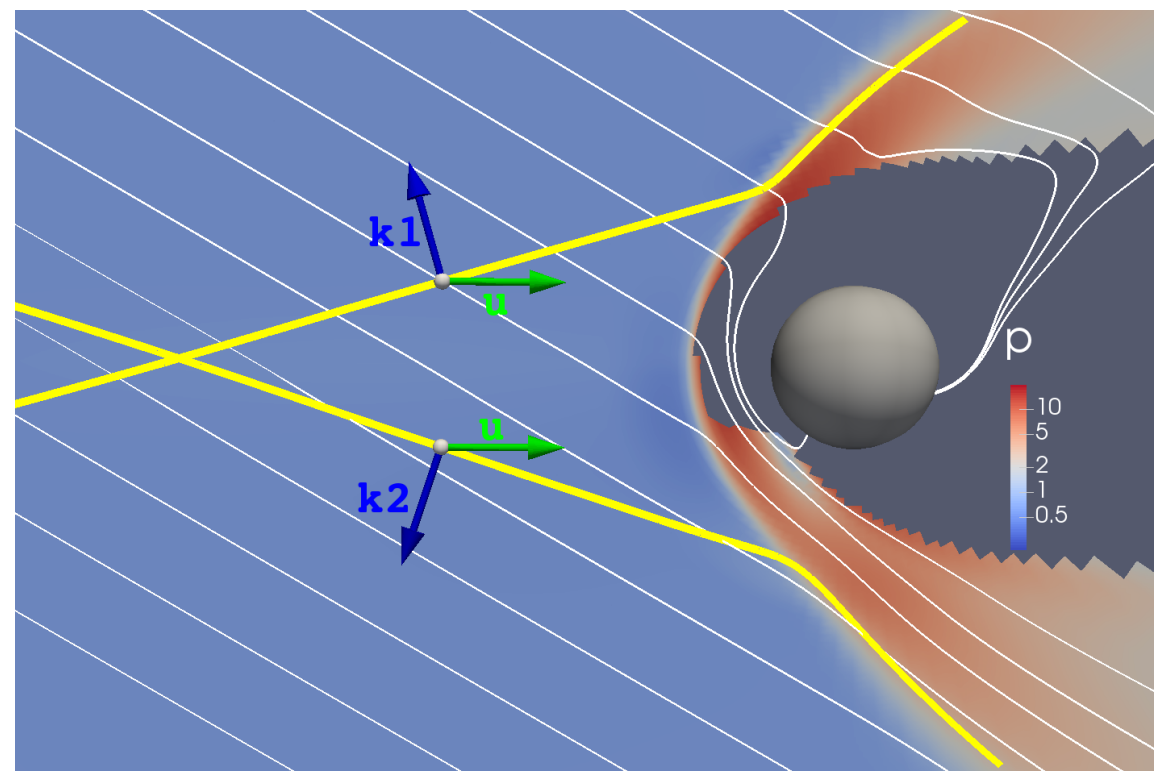

Fig. 12.- Color coded contours of the thermal pressure $p$ in a simulation of the interaction of the solar wind with Mercury's magnetosphere. Magnetic field lines are in white. The yellow curves represent two possible standing fast mode solutions given by equation (30). The regions with no pressure contours are those where no standing fast modes are possible $(D<0$ in (30)).

Under such circumstances, as previously explained for Figure 1, for any given point in the plane there are at most two standing fronts with wave vectors $\vec{k}_{1,2}$. The corresponding orientations $\theta_{1,2}$ are given by equation (30) with $\varphi$ either 0 or $\pi$, the $y$ components of all vectors being necessarily zero in the plane of Figure 12. In the free solar wind (on the left in Fig. 12) there are no waves and the two fronts do merely show a cut through the Mach cone which would be generated by a point obstacle placed at the crossing of the two fronts. Accordingly, the two fronts do also represent the orientation of the bow shock at a large distance from the planet and more generally equation (30) fully describes the fast Mach cone generated by a point obstacle moving through the plasma at speed $\vec{u}_{f}$. The fast Mach cone defined by equation (30) is an approximation but far better handleable than the exact form given in Verigin et al. (2003).

\subsection{Sample application for the slow mode}

For the slow mode case discussed in section 4 we consider the particular problem of the formation of a slow mode expansion fan at the Jovian moon Io as described by Krisko and Hill (1991). 


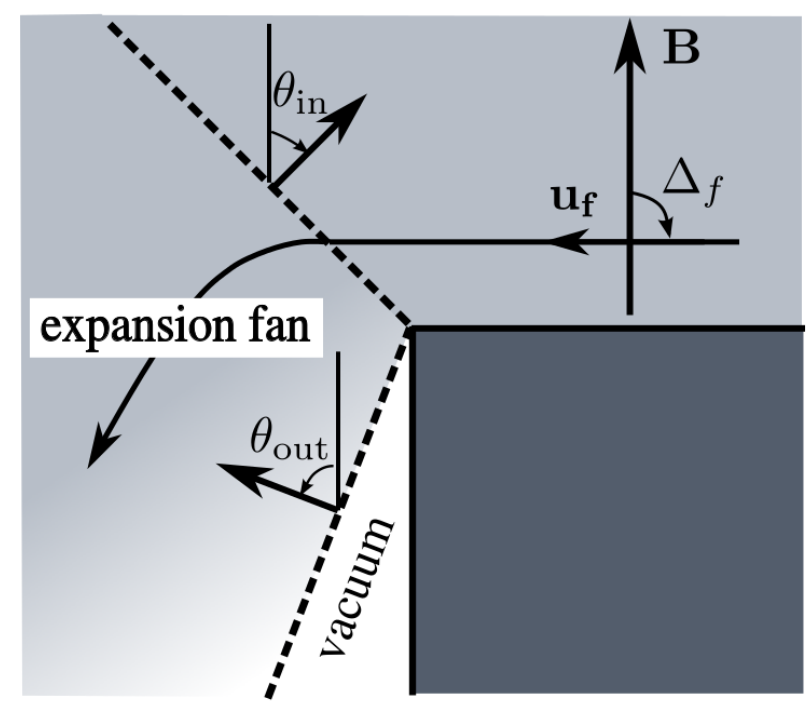

Fig. 13. - Schematic illustration of the problem of a plasma flowing over a nonconducting twodimensional sharp corner leading to the formation of a slow mode expansion fan. The angle $\theta_{i n}$ denotes the orientation of the leading standing slow mode front, marking the entrance of the fan. The angle $\theta_{\text {out }}$ denotes the front separating the plasma from the vacuum.

Following Siscoe and Sanchez (1987), Krisko and Hill solve a simplified version of the problem which is that of a steady state plasma flowing over a nonconducting, two-dimensional sharp corner as illustrated in Fig. 13. The problem is self-similar as it is free of any characteristic spatial scale. As a consequence, plasma parameters do only vary as a function of the azimuthal angle and do not depend on the distance (both angles and distances are measured with respect to the corner). We note that unlike Siscoe and Sanchez (1987) who were interested in the high beta environment of the Earth magnetosphere (NB: $a^{2}=2 /(\gamma \beta)$ ), Krisko and Hill (1991) solve a low beta case (see Table 1) where the magnetic field is essentially unaffected by the flow.

Table 1: Orientation of the standing slow modes delimiting the expansion fan in the case of a plasma flowing over a sharp corner for the particular case treated by Krisko and Hill (1991). $\theta_{\mathrm{KH}}$ are the orientations given by Krisko and Hill, $\theta_{\text {app }}$ have been computed using the approximation (18)

\begin{tabular}{l|c|c|c|c|c|c}
\hline Parameter & $a$ & $u_{f}$ & $\Delta_{f}[\mathrm{deg}]$ & $\theta_{\mathrm{KH}}[\mathrm{deg}]$ & $\theta_{\mathrm{app}}[\mathrm{deg}]$ & $\delta \theta[\mathrm{deg}]$ \\
\hline In & 6.67 & 1.0 & 90.0 & 45.0 & 44.36 & 0.18 \\
Out & 2780 & 27.8 & 20.0 & 159 & 159.27 & $\epsilon$ \\
\hline
\end{tabular}

Solution S2 (equation (18)) is the one compatible with the orientation of the magnetic field in Fig. 13. As shown in Table 1, the approximate orientations $\theta_{\text {app }}$ of the slow mode fronts defining the expansion fan are nearly identical to those given by Krisko and Hill (1991). This is not surprising, 
as the parameter $a$ is substantially larger than unity, in which case equation (18), which has been used to compute $\theta_{\text {app }}$, provides an excellent approximation.

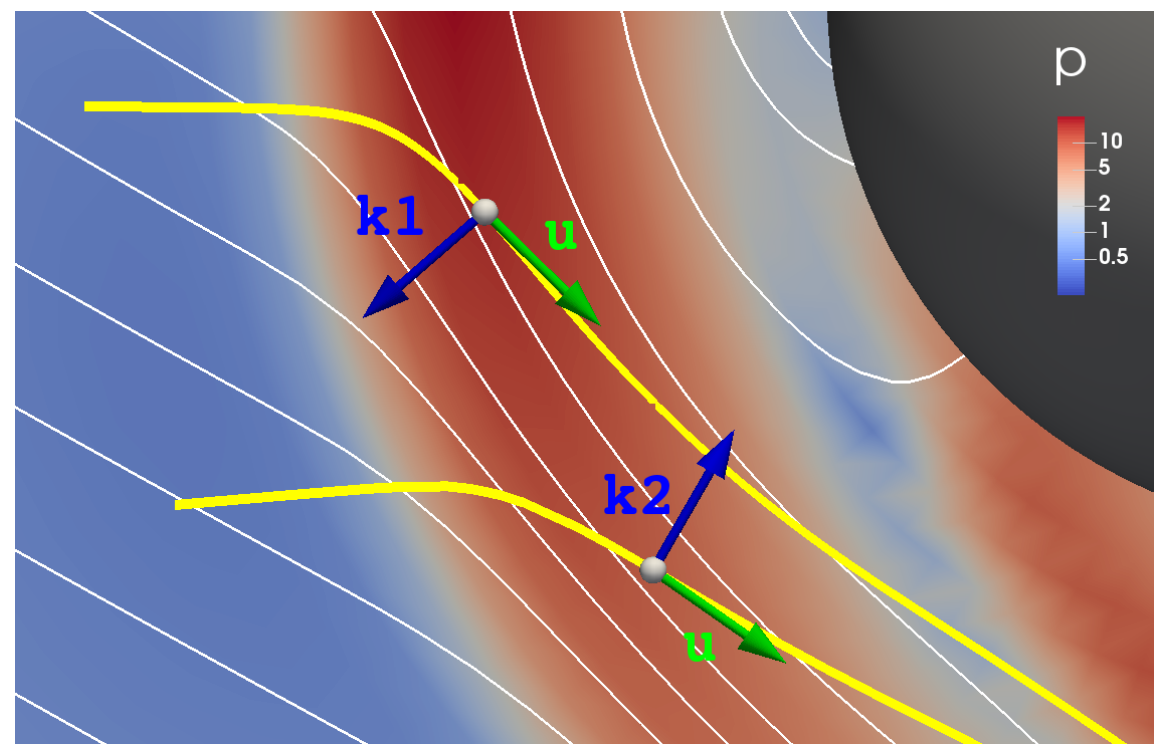

Fig. 14.- Cut through a three-dimensional MHD simulation of Mercury's magnetosphere. The plasma flows horizontally from left to right and goes through a fast mode shock visible as a sharp rise of the pressure $p$. The thin white lines are magnetic field lines while the thick yellow lines are two examples of possible standing slow mode fronts propagating nearly opposite to each other and representing the two solutions of Figure 1 with wave vector $\vec{k}_{1}$ and $\vec{k}_{2}$, respectively.

As suggested by Pantellini et al. (2015), standing slow mode fronts possibly form downstream of planetary bow shocks (the magnetosheath) where the plasma flow velocity vector and the magnetic field vector are nearly parallel to each other (i.e. $\Delta_{f} \ll 1$ ). Figure 14 shows a portion of the same cut through the 3D simulation by Pantellini et al. (2015) already shown in Figure 12. As for the fast mode in Figure 12, standing wave fronts with their associated wave vectors and local flow velocity vectors at two selected points are shown for the slow mode. The two fronts have been constructed using the expressions (17) and (18) with $\varphi$ either 0 or $\pi$. It is unlikely that both fronts, roughly propagating in opposite directions, coexist with similar amplitudes in any given region of space as their energy source regions are necessarily different. For the slow mode, energy propagates essentially along the magnetic field line. In the region of the magnetosheath shown in Figure 14, it is more likely that a hypothetical slow mode front is rather of the $\vec{k}_{1}$ sustained by an energy flow from the nose region of the magnetosheath where plasma flow deflection and magnetic field lines pile-up are strongest.

A slow mode structure can be distinguished from a fast mode structure based on measurements of the parallel compressibility $C_{\|}=\delta n B /\left(n \delta B_{\|}\right)$, where $\delta n$ and $\delta B_{\|}$represent the spatial variations normal to the wave front, i.e. along the direction given by the wave vector. Indeed, as shown in Appendix B, the parallel compressibility is positive for the fast mode and negative for the slow 

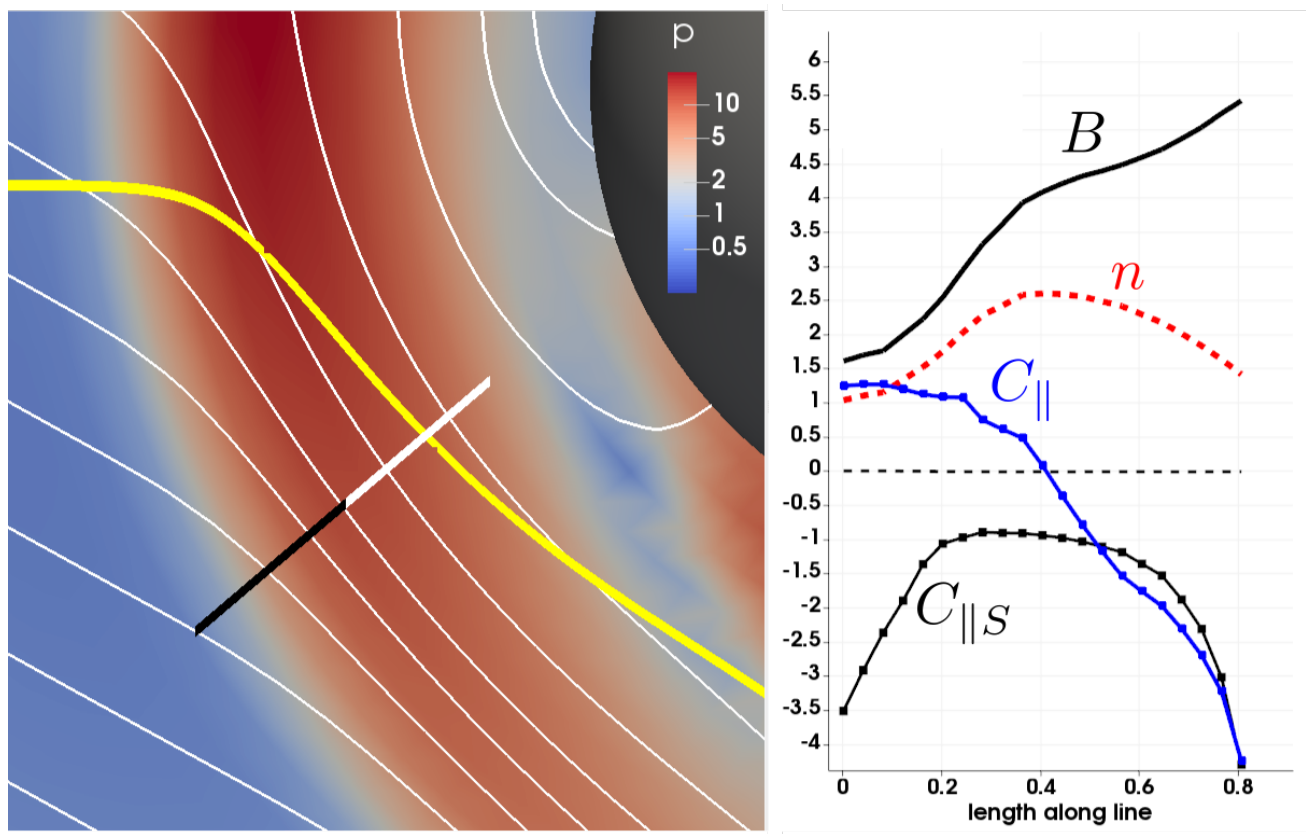

Fig. 15. - Left panel: same format as Figure 14 with only the $\vec{k}_{1}$ front shown. Right panel: plasma density $n$, magnetic field intensity $B$, measured parallel compressibility $C_{\|}$and theoretical parallel compressibility for the slow mode $C_{\| S}$ along the thick straight line shown in the left panel. Note how the measured compressibility $C_{\|}$turns negative and approaches $C_{\| S}$ after position 0.4 suggesting a transition from a fast mode dominated to a slow mode dominated structure.

mode. Thus, by comparing the measured compressibility with the theoretical predictions for the various modes along a direction normal to the density and/or magnetic field gradients may allow identifying the local dominant mode. As an example, the parallel compressibility measured along a straight line aligned with the wave vector $\vec{k}_{1}$ is shown in the right panel of Figure 15 . As expected, the $C_{\|}$profile is positive near the shock front (the bow shock being a fast mode shock). Deeper inside the magnetosheath at the density maximum at position $0.4, C_{\|}$turns negative and approaches the theoretical prediction $C_{\| S}$ for the slow mode, suggesting that the fast mode is no longer the dominant mode.

\section{Conclusion}

We have presented analytic expressions describing standing plane modes in a steady state plasma flow for the three linear MHD modes. Expressions are exact for the Alfvén mode and approximate for fast and the slow modes. They provide comparatively simple analytic forms of the Alfvén, slow and fast Mach cones. The can be used as a valuable tool to help identify the dominant modes in complex experimental or simulated plasma flows. 
The work of Léa Griton was supported by the Centre National d'Études Spatiales (CNES) and the Observatoire de Paris (contract ref. 5100016058). Part of the presented work has been completed by Laura Munteanu during a one-month internship at LESIA in June 2015.

\section{A. Changing reference frame}

The calculations presented in this paper have been established in a particular reference frame (hereafter the primed reference frame) defined by the flow velocity vector $\vec{u}_{f}$ and the magnetic field vector $\vec{B}$ as illustrated in Figure 1. This particular frame is well suited for the theoretical treatment of mode propagation related issues but is rarely the most practical one as both velocity and magnetic field orientations do generally change as a function of space. In most applications, however, there is one unique frame for the whole system (for example the simulation frame as in Fig. 14 or the frame defined by the step geometry in the Krisko and Hill 1991 experiment of Fig. 13). We denote this frame as the unprimed frame. It is therefore useful to establish the matrix $M$ which transforms the orthonormal right handed basis vectors of an arbitrary frame into the orthonormal basis vectors (also right handed) of the primed reference frame. The plasma flow being stationary in both frames there is no relative motion and the transformation matrix $M$ may be viewed as the product of two rotations implying $\operatorname{det}(M)=1$. $M$ is easily obtained by writing the basis vectors $\vec{e}_{x}{ }^{\prime}, \vec{e}_{y}{ }^{\prime}$ and $\vec{e}_{z}{ }^{\prime}$ of the primed reference frame in terms of the two unitary vectors $\vec{\mu}_{f} \equiv \vec{u}_{f} / u_{f}$ and $\vec{b} \equiv \vec{B} / B$ in the unprimed frame, i.e.

$$
\begin{aligned}
& \vec{e}_{z}{ }^{\prime}=-s \vec{b} \\
& \vec{e}_{y}{ }^{\prime}=\frac{\vec{\mu}_{f} \times \vec{e}_{z}{ }^{\prime}}{\sin \Delta_{f}}=-s \frac{\vec{\mu}_{f} \times \vec{b}}{\sin \Delta_{f}} \\
& \vec{e}_{x}{ }^{\prime}=\vec{e}_{y}{ }^{\prime} \times \vec{e}_{z}{ }^{\prime}=s \frac{\left(\vec{\mu}_{f} \times \vec{b}\right) \times \vec{b}}{\sin \Delta_{f}}=\frac{-\vec{\mu}_{f}+\left(\vec{\mu}_{f} \cdot \vec{b}\right) \vec{b}}{\sin \Delta_{f}}
\end{aligned}
$$

where $s=\operatorname{sign}\left(\vec{\mu}_{f} \cdot \vec{b}\right)$ and $0<\Delta_{f} \leq \pi / 2$ is the angle between the directions of $\vec{b}$ and $\vec{\mu}_{f}$. In the primed frame the magnetic field is therefore either parallel or anti parallel with respect to $\vec{e}_{z}{ }^{\prime}$. If $\Delta_{f} \neq 0$, the components of the three primed basis vectors are fully specified by the constraint that $\vec{\mu}_{f}$ is in the $\left(\vec{e}_{x}{ }^{\prime}, \vec{e}_{z}{ }^{\prime}\right)$ plane with negative components $\mu_{f x}{ }^{\prime}=\vec{\mu}_{f} \cdot \vec{e}_{x}{ }^{\prime}<0$ and $\mu_{f z}{ }^{\prime}=\vec{\mu}_{f} \cdot \vec{e}_{z}{ }^{\prime}<0$. In the singular case $\Delta_{f}=0, \vec{e}_{y}{ }^{\prime}$ may be any unitary vector perpendicular to $\vec{b}$ and $\vec{e}_{x}{ }^{\prime}=\vec{e}_{y}{ }^{\prime} \times \vec{e}_{z}{ }^{\prime}$ as in the general case (A1).

The transformation of the basis vectors $\vec{e}_{x}=(1,0,0), \vec{e}_{y}=(0,1,0)$ and $\vec{e}_{z}=(0,0,1)$ to the primed frame is thus given by

$$
\vec{e}_{i}{ }^{\prime}=M \vec{e}_{i}, i=\{x, y, z\}
$$

where the elements of the $3 \times 3$ transformation matrix $M$ are merely the components of the primed basis vectors:

$$
M=\left(\vec{e}_{x}{ }^{\prime}\left|\vec{e}_{y}{ }^{\prime}\right| \vec{e}_{z}{ }^{\prime}\right) .
$$


Accordingly, the components of an arbitrary vector projected onto the basis vectors of the primed frame are obtained by applying the transposed matrix $M^{T}$ to the components of the vector in the unprimed frame, i.e.

$$
\vec{v}^{\prime}=M^{T} \vec{v}
$$

where $\vec{v}^{\prime}=\left(\vec{v} \cdot \vec{e}_{x}{ }^{\prime}, \vec{v} \cdot \vec{e}_{y}{ }^{\prime}, \vec{v} \cdot \vec{e}_{z}{ }^{\prime}\right)$.

\section{A.1. Special case: $\mu_{f y}=b_{y}=0$}

The transformation matrix $M$ is particularly simple in the case where the $y$ components of $\vec{\mu}_{f}$ and $\vec{b}$ are zero in the unprimed frame. In this case the basis vectors (A1) reduce to:

$$
\begin{aligned}
& \vec{e}_{z}^{\prime}=-s\left(b_{x}, 0, b_{z}\right) \\
& \vec{e}_{y}^{\prime}=-s(0, \sigma, 0) \\
& \vec{e}_{x}{ }^{\prime}=+\sigma\left(b_{z}, 0,-b_{x}\right)
\end{aligned}
$$

where $\sigma \equiv \operatorname{sign}\left(-\mu_{f x} b_{z}+\mu_{f z} b_{x}\right)$. Accordingly, the transformation matrix $M$ for this particular case is

$$
M=\left(\begin{array}{ccc}
\sigma b_{z} & 0 & -s b_{x} \\
0 & -s \sigma & 0 \\
-\sigma b_{x} & 0 & -s b_{z}
\end{array}\right)
$$

\section{B. Parallel compressibility and Alfvén ratio}

\section{B.1. The parallel compressibility}

The parallel compressibility of a plane mode with wave vector $\vec{k}$ is defined as

$$
C_{\|}=\frac{\delta n}{n} \frac{B}{\delta B_{\|}}
$$

where $\delta n$ and $\delta B_{\|}$are the variations of density and magnetic field along a path parallel to $\vec{k}$. The subscript $\|$ in $\delta B_{\|}$denotes the variation of the magnetic field parallel to itself so that (for example) $\delta B_{\|}=0$ through a rotational discontinuity. Assuming a wave vector $\vec{k}=(k, 0,0)$ :

$$
\delta B_{\|}=d x \frac{\partial \vec{B}}{\partial x} \cdot \frac{\vec{B}}{B}=d x \frac{\partial B}{\partial x}
$$

and

$$
\delta n=d x \frac{\partial n}{\partial x}
$$


In case of an arbitrary orientation $\vec{k}$ the variations $\delta B_{\|}$and $\delta n$ over an infinitesimal distance $\overrightarrow{\delta x}=\epsilon \vec{k}$ are

$$
\delta B_{\|}=\overrightarrow{\delta x} \cdot \nabla B=\epsilon \vec{k} \cdot \nabla B
$$

and

$$
\delta n=\epsilon \vec{k} \cdot \nabla n
$$

respectively. The parallel compressibility can then be written as:

$$
C_{\|}=\frac{\vec{k} \cdot \nabla n}{n} \frac{B}{\vec{k} \cdot \nabla B}
$$

For the incompressible Alfvén mode the parallel compressibility is zero. For both the slow and the fast mode the parallel compressibility is given by

$$
C_{\|}(\theta)=\frac{c_{A}^{2}}{u_{\phi}^{2}(\theta)-c^{2}}
$$

where $\theta$ is the angle between $\vec{k}$ and the magnetic field $\vec{B}, c_{A}$ is the Alfvén speed, $c$ the adiabatic sound speed and $u_{\phi}=\omega / k$ the phase velocity (3) of the corresponding mode. We note that for non-zero values of the Alfvén speed, the fast mode does always propagate faster than the sound speed. Thus, according to (B7) the compressibility of the fast mode is always positive. On the contrary, slow modes do always propagate slower than the sound speed (except for the special case $c=c_{A}$ and $\theta=0$ ). Thus, the denominator in (B7) is always negative implying a negative compressibility for the slow mode. A typical example of parallel compressibility profiles for both compressible MHD modes is shown in Fig. 16.

\section{B.2. The Alfvén ratio}

The Alfvén ratio is defined as

$$
R_{A}=\frac{\delta v_{\perp, k}^{2}}{c_{A}^{2}} \frac{B^{2}}{\delta B_{\perp, k}^{2}}
$$

where the perpendicular direction is now to be considered with respect to $\vec{k}$, i.e $\delta v_{\perp, k}=|\overrightarrow{\delta v} \times \vec{k} / k|$.

For the three MHD modes the Alfvén ratio is given by

$$
R_{A}(\theta)=\frac{c_{A}^{2}}{u_{\phi}^{2}} \cos ^{2} \theta .
$$

The phase velocity of the Alfvén mode being $u_{\phi}^{2}=c_{A}^{2} \cos ^{2} \theta$ implies that $R_{A}=1$, independently of the propagation angle $\theta$. Sample profiles of the Alfvén ratio for the three MHD modes are shown in Fig. 16. 


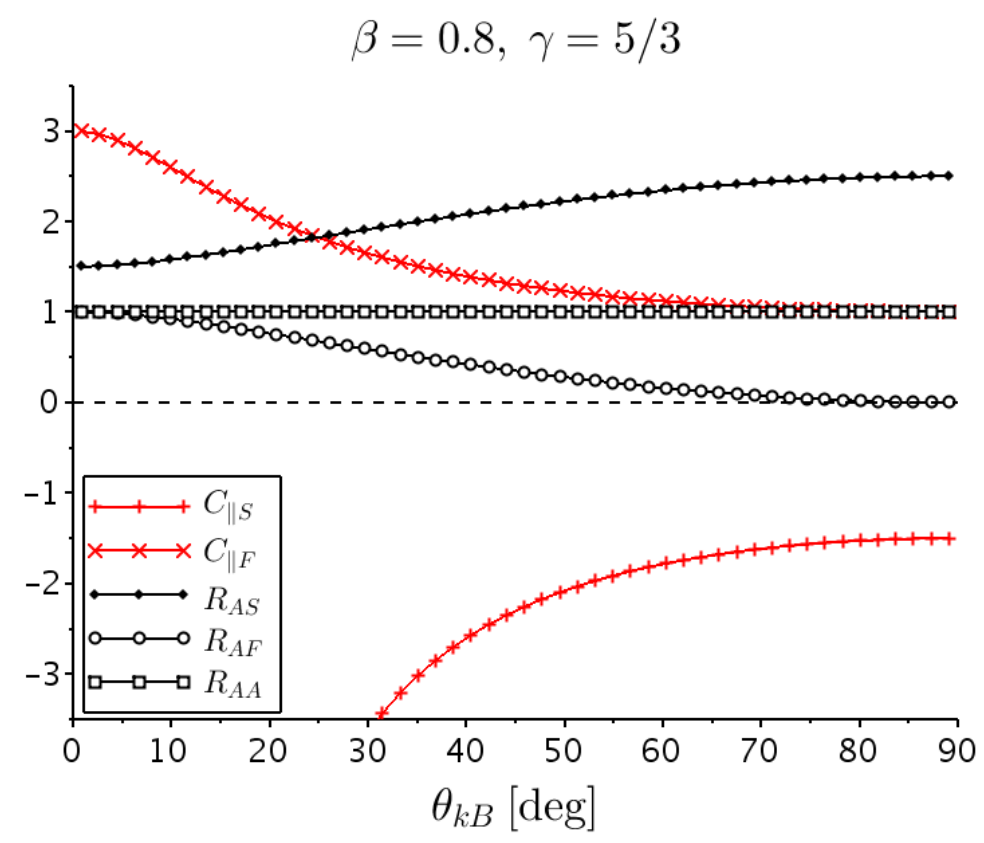

Fig. 16. - Parallel compressibility and Alfvén ratio for the 3 MHD modes and particular values of $\beta$ and $\gamma$.

\section{REFERENCES}

P. H. Krisko and T. W. Hill. Two-dimensional model of a slow-mode expansion fan at Io. Geophysical Research Letters, 18:1947-1950, November 1991. doi: 10.1029/91GL02541.

C. Lacombe, F. G. E. Pantellini, D. Hubert, C. C. Harvey, A. Mangeney, G. Belmont, and C. T. Russell. Mirror and Alfvenic waves observed by ISEE 1-2 during crossings of the earth's bow shock. Annales Geophysicae, 10:772-784, October 1992.

F. Pantellini, L. Griton, and J. Varela. Rarefaction and compressional standing slow mode structures in Mercury's magnetosheath: 3D MHD simulations. Planetary and Space Science, 112: 1-9, July 2015. doi: 10.1016/j.pss.2015.04.007.

G. L. Siscoe and E. Sanchez. An MHD model for the complete open magnetotail boundary. J. Geophys. Res., 92:7405-7412, July 1987. doi: 10.1029/JA092iA07p07405.

J. R. Spreiter and A. Y. Alksne. Solar-wind flow past objects in the solar system. Annual Review of Fluid Mechanics, 2:313-354, 1970. doi: 10.1146/annurev.fl.02.010170.001525.

M. Verigin, J. Slavin, A. Szabo, G. Kotova, and T. Gombosi. Planetary bow shocks: Asymptotic MHD Mach cones. Earth, Planets, and Space, 55:33-38, January 2003. 
This preprint was prepared with the AAS $\mathrm{LAT}_{\mathrm{E}} \mathrm{X}$ macros v5.2. 